INPLASY

PROTOCOL

To cite: Zhang et al.

Comparison of the

effectiveness of different

acupuncture methods in

relieving cancer pain:

systematic review and network

meta-analysis of randomised

controlled trials. Inplasy

protocol 202210127. doi:

10.37766/inplasy2022.1.0127

Received: 27 January 2022

Published: 27 January 2022

Corresponding author:

Jingwen Zhang

zhangjingwen828@163.com

Author Affiliation:

Jiangxi University of Chinese

Medicine

Support: Jiangxi Province, China.

Review Stage at time of this submission: Preliminary

searches.

Conflicts of interest:

None declared.

\section{Comparison of the effectiveness of different acupuncture methods in relieving cancer pain: systematic review and network meta-analysis of randomised controlled trials}

Zhang, Q1; Huang, S2; Wu, Q3; Jiang, H4; Huang, S5; Zhang, J6.

Review question / Objective: Objectives: In this paper, the efficacy of different acupuncture therapies in alleviating pain of patients with cancer will be systematically evaluated and network meta-analyzed, and the safety of acupuncture therapy will be evaluated, which can help guide the clinical application with scientific methods in the future. Participants: patients with cancer suffering from pain, including pain caused by the development of cancer and / or chronic pain caused by cancer treatment . Interventions: Acupuncture can be adopted for intervention, combining analgesic therapy or routine nursing. Comparisons: treatments adopted in the control group consists of sham acupuncture, placebo acupuncture, analgesic treatment, routine nursing, etc., without additional intervention. Outcome: the intensity of pain was chosen as the target outcome, the Brief Pain Inventory, Numerical Rating Scale, Visual Analog Scale, Verbal Rating Scale, and other validated instruments were adopted for assessing the intensity of pain. Study design:Clinical random control trials.

INPLASY registration number: This protocol was registered with the International Platform of Registered Systematic Review and Meta-Analysis Protocols (INPLASY) on 27 January 2022 and was last updated on 27 January 2022 (registration number INPLASY202210127).

\section{INTRODUCTION}

Review question / Objective: Objectives: In this paper, the efficacy of different acupuncture therapies in alleviating pain of patients with cancer will be systematically evaluated and network meta-analyzed, and the safety of acupuncture therapy will be evaluated, which can help guide the clinical application with scientific methods in the future. Participants: patients with cancer suffering from pain, including pain caused by the development of cancer and / or chronic pain caused by cancer treatment . 
Interventions: Acupuncture can be adopted for intervention, combining analgesic therapy or routine nursing. Comparisons: treatments adopted in the control group consists of sham acupuncture, placebo acupuncture, analgesic treatment, routine nursing, etc., without additional intervention. Outcome: the intensity of pain was chosen as the target outcome, the Brief Pain Inventory, Numerical Rating Scale, Visual Analog Scale, Verbal Rating Scale, and other validated instruments were adopted for assessing the intensity of pain. Study design:Clinical random control trials.

Condition being studied: In this research, manual and computer aided search methods were adopted, the database includes CNKI, WANFANG, VIP, SinoMed, PubMed, Cochrane Library, Embase, and the content of the research is the clinical random control of different acupuncture therapies in the treatment of cancer pain Trials (RCTs), the search period is from the establishment of the database to January 24, 2022, in addition, literature was managed and screened by EndNote software; the risk bias was assessed using the Cochrane tool; the results of the these papers were analyzed using the stata 17 software.

\section{METHODS}

Search strategy: In this research, manual and computer aided search methods were adopted, the database includes CNKI, WANFANG, VIP, SinoMed, PubMed, Cochrane Library, Embase, and the content of the research is the clinical random control of different acupuncture therapies in the treatment of cancer pain Trials (RCTs), the search period is from the establishment of the database to January 24, 2022.

Participant or population: Patients with cancer suffering from pain, including pain caused by the development of cancer and / or chronic pain caused by cancer treatment.
Intervention: The qualified measures for intervention are acupuncture, including traditional acupuncture, electroacupuncture, warm acupuncture, etc, which can be combined with routine analgesic therapy or routine nursing.

Comparator: sham acupuncture or placebo acupuncture and analgesic treatment or routine care, no additional intervention measures are allowed.

Study designs to be included: Randomized controlled trials (RCTs) will be included.

Eligibility criteria: Criteria for inclusion: 1. Clinical random control trials. 2. Patients with cancer pain, including Pain directly accompanying the development of cancer and/or chronic pain associated with cancer treatments was included. 3. Measures of intervention include various acupuncture methods. 4. The control measures were sham acupuncture, analgesic therapy, routine nursing, etc. There is no additional intervention such as traditional Chinese medicine, moxibustion, etc.Exclusion criteria: 1 The study of random grouping method was not reported in detail. 2 . Unable to obtain the full text of the research. 3. Studies that have been withdrawn 4. Grey researches 5. Only efficient studies were reported. 6. Studies of acupuncture for short-term analgesia associated with surgical procedures were excluded.

Information sources: In this research, manual and computer aided search methods were adopted, the database includes CNKI, WANFANG, VIP, SinoMed, PubMed, Cochrane Library, Embase, and the content of the research is the clinical random control of different acupuncture therapies in the treatment of cancer pain Trials (RCTs), the search period is from the establishment of the database to January 24, 2022.

Main outcome(s): In terms of outcome indicators, patients' pain scores, such as Brief Pain Inventory, Numerical Rating Scale, Visual Analog Scale, Verbal Rating 
Scale, and other validated instruments for assessing the intensity of pain.

Quality assessment / Risk of bias analysis: Two review authors will assess the risk of bias of each individual included study based on methods endorsed by The Cochrane Collaboration. The following domains will be considered: randomization, allocation concealment, blinding, selective reporting, publication bias, as well as any other detected sources of bias that may arise. The risk of bias will be assessed at study level. However, the risk of bias of each study will be considered when we conduct the summary of findings table.

Strategy of data synthesis: We carried out a frequentist network meta-analysis. Risk ratio (RR) with $95 \%$ confidence interval (Cl) for outcomes with $95 \%$ CI were summarized. The ranking probabilities for all treatments of being at each possible rank for each intervention were estimated. To check the assumption of consistency in the entire analytical network, a design-bytreatment approach was used. Global heterogeneity was assessed with the 12statistic which incorporates the extent of heterogeneity and was used to evaluate the extent of uncertainty in the estimated effect size locally. Comparison-adjusted funnel plots were performed to investigate whether the integrated results have differences between imprecise trials and precise trials. The relative ranking probabilities for each acupuncture therapy and the surface under the cumulative ranking (SUCRA) were calculated to determine the relative effect of each acupuncture therapy. All analyses were conducted using stata17.0 and R 3.6.2.

Subgroup analysis: Not applicable.

Sensitivity analysis: Not applicable.

Country(ies) involved: China.

Keywords: acupuncture, cancer pain, network meta-analysis, randomizedcontrolled trials.
Contributions of each author:

Author 1 - Qingyuan Zhang. Email: zqy1072025995@163.com

Author 2 - Shenghe Huang.

Author 3 - Qianyan Wu.

Author 4 - Hongliang Jiang.

Author 5 - Shuailiang Huang.

Author 6 - Jingwen Zhang.

Email: zhangjingwen828@163.com 